\title{
Ethnic differences in retinal microvascular structure
}

\author{
T. Tillin • R. M. Evans • N. W. Witt • P. S. Sharp • \\ P. M. McKeigue $\cdot$ N. Chaturvedi $・$ A. D. Hughes
}

Received: 9 May 2008 / Accepted: 12 June 2008 / Published online: 15 July 2008

(C) Springer-Verlag 2008

\begin{abstract}
Aims/hypothesis People of African origin have increased risk of stroke and retinal microvascular disease compared with populations of European origin. We compared quantitative measures of retinal microvasculature in British white Europeans and African Caribbeans.

Methods Population-based study of 215 (45\% male) British African-Caribbean migrants and 323 (48\% male) white Europeans aged 40-69 years. Digitised retinal images were analysed using a validated semi-automated system.

Results Arteriolar optimality deviation, an indicator of endothelial dysfunction, was greater in African Caribbeans (age- and sex-adjusted means [95\% CIs]: 0.06 [0.05-0.06] vs 0.04 [0.04-0.05], $p=0.004$ ); this was unexplained by conventional risk factors. Arteriolar diameters were narrower in African Caribbeans (age- and sex-adjusted means [95\% CIs]: 18.4 [18.1-18.6] vs 17.9 [17.6-18.2], $p=0.011$ ). These ethnic differences in diameters were attenuated on adjustment for systolic BP (SBP) (adjusted means: 18.2 vs $18.1, p=0.31$ ). However, there was a significant interaction
\end{abstract}

T. Tillin $(\bowtie) \cdot$ R. M. Evans $\cdot$ N. W. Witt $\cdot$ N. Chaturvedi

A. D. Hughes

International Centre for Circulatory Health,

Imperial College London,

59 North Wharf Road,

London W2 1LA, UK

e-mail: t.tillin@imperial.ac.uk

P. S. Sharp

Department of Diabetes and Endocrinology, Southampton General

Hospital,

Southampton, UK

P. M. McKeigue

MRC Human Genetics Unit, University of Edinburgh,

Edinburgh, UK
( $p=0.011)$ between diabetes and SBP, such that SBP was strongly associated with arteriolar diameter in people without diabetes, but not in those with diabetes (adjusted $\beta$-coefficients for SBP: Europeans: $-0.42, p=0.002$ vs 0.17 , $p=0.69$, African Caribbeans: $-0.35, p=0.023$ vs $0.01, p=0.96$ ). Other measures of retinal vasculature did not differ by ethnicity.

Conclusions/interpretation British African Caribbeans appear to have poorer retinal arteriolar endothelial function than white Europeans. Higher BPs explained the narrower arterioles in African Caribbeans; however, patterns of association between arteriolar narrowing and BP suggest the possibility that cerebral autoregulation and/or remodelling might be adversely affected by diabetes in both ethnic groups.

Keywords Blood pressure - Diabetes - Ethnicity Microcirculation $\cdot$ Retina
Abbreviations
LDR length:diameter ratio
SBP systolic BP

\section{Introduction}

People of African origin worldwide have an increased risk of cerebrovascular disease, the risk being about 1.1- to 2.3fold that of populations of European origin in the UK [1]. This increased risk of stroke is associated with increased small vessel pathology [2]. Measurable microvascular diseases such as retinopathy [3] are similarly elevated in people of African origin. These observations have been thus far unexplained by conventional risk factors. 
The retinal microvasculature provides a readily accessible site for non-invasive measurement of a microcirculatory bed that shares functional properties with the cerebral circulation.

We have previously demonstrated associations between retinal arteriolar narrowing and hypertension [4] and between arteriolar bifurcation optimality and lower-limb atherosclerosis [5] and ischaemic heart disease mortality [6].

We hypothesised that retinal arteriolar bifurcation properties and arteriolar diameters would be more adverse in British people of African-Caribbean origin than in Europeans and that ethnic differences would be explained by diabetes or BP.

\section{Methods}

Participants, baseline measurements and retinal photography We performed new analyses of retinal photographs collected during the baseline phase (1990-1991) of a population-based bi-ethnic cohort of 578 first-generation British African Caribbeans and 582 white Europeans aged 40-69 years (the Brent Study) [7]. Local ethical committee approval was granted and all participants gave written informed consent.

The response rate was $58 \%$. All participants $(n=693)$ who attended one centre, which had photography facilities, were eligible for retinal photography. Participants had $45^{\circ}$ macular field photographs of each eye taken using a nonmydriatic camera after dilatation of the pupil with $1 \%$ tropicamide.

Participants completed a questionnaire on medical history and lifestyle. Anthropometrics and sitting BP were recorded. Fasting blood samples were taken. World Health Organization 1999 criteria were used to define diabetes.

Retinal microvascular analyses Digitised images were analysed using a custom-written semi-automated program running within the Matlab programming environment. Diameters were measured to sub-pixel resolution. Vessel length was tracked along the vessel between bifurcations. The length:diameter ratio (LDR) is insensitive to the refractive properties of the eye and was calculated as the ratio of the length of a vessel segment to its average diameter between two branching points.

We assessed the extent to which arteriolar bifurcations depart from the optimal diameter relationship predicted by Murray's law [8], by calculating the ratio of the mean diameter of daughter vessels to the diameter of the parent vessel. After correction for bifurcation asymmetry, a bifurcation compliant with Murray's law has an asymmetry ratio of 0.79 (cube root of 1/2) [8], and the absolute amount by which the measured ratio differs from this value is known as the optimality deviation. An optimal bifurcation experiences uniform shear stress, and deviation from optimum indicates endothelial dysfunction [9].

Images were graded by two trained observers, blinded to participant identity and ethnicity. Bland-Altman coefficients of repeatability (inter-observer) for arteriolar length diameter ratio and optimality ratio were 5.2 and 0.16 .

Statistical analyses Retinal vasculature measurements were examined in each ethnic group. Skewed variables were appropriately transformed. People receiving treatment for hypertension were allocated to the top rank/tertiles of BP or separately categorised. Interactions between ethnicity and each risk factor were assessed. There were no significant interactions between ethnicity and sex for any retinal measurements; hence data are not stratified by sex. Ethnic group differences in retinal measurements were explored in linear regression models; age, sex, ranked systolic BP (SBP) and fasting glucose were forced into multivariate models.

\section{Results}

Baseline characteristics Of the 683 sets of retinal images, 28\% (84/299) African Caribbean and 16\% (61/384) European were not analysable because of poor image quality. Those with analysable retinal images consisted of 215 African Caribbeans and 323 Europeans. Measures of dysglycaemia and hypertension were worse in African Caribbeans compared with Europeans (Table 1). Findings in people with analysable retinal images were consistent with the baseline characteristics of the whole study population.

Retinal vascular measurements Median numbers of arterioles and arteriolar bifurcations analysed were seven and five, respectively; $79 \%$ were graded in one eye only, because of image quality. The numbers of images per person analysed did not differ by ethnicity.

African Caribbeans had greater deviation from optimal diameter relationships at arteriolar bifurcations compared with Europeans (Table 1). Ethnic group differences in optimality deviation did not vary by diabetes, hypertension or metabolic syndrome status and were unexplained by measured risk factors (Table 1).

African Caribbeans had narrower arterioles than Europeans, and correspondingly greater arteriolar LDRs. These ethnic differences remained when we excluded those receiving treatment for hypertension, but were attenuated on adjustment for SBP (Table 1). 
Table 1 Characteristics of those with analysable retinal images

\begin{tabular}{|c|c|c|c|}
\hline Characteristics & White Europeans & African Caribbeans & $p$ value \\
\hline No. with analysable images & 323 & 215 & \\
\hline Age (years) & $52.6 \pm 6.5$ & $53.3 \pm 5.9$ & 0.030 \\
\hline Male sex, \% $(n)$ & $48(154)$ & $45(96)$ & 0.51 \\
\hline Smoking, \% (current/ex-/never) & $38 / 26 / 36$ & $22 / 15 / 64$ & $<0.001$ \\
\hline Diabetes (known or newly diagnosed), \% ( $n)$ & $9(30)$ & $24(51)$ & $<0.001$ \\
\hline Treated hypertension, $\%(n)$ & $8(27)$ & $25(53)$ & $<0.001$ \\
\hline $\mathrm{SBP}^{\mathrm{a}}(\mathrm{mmHg})$ & $120(113,126)$ & $133(123,147)$ & $<0.001$ \\
\hline $\mathrm{DBP}^{\mathrm{a}}(\mathrm{mmHg})$ & $76(72,81)$ & $85(79,98)$ & $<0.001$ \\
\hline Fasting glucose $(\mathrm{mmol} / \mathrm{l})$ & $5.7(5.2,6.2)$ & $5.8(5.3,6.5)$ & $<0.001$ \\
\hline \multicolumn{4}{|l|}{ Retinal variables adjusted for age and $\operatorname{sex}^{\mathrm{b}}$} \\
\hline Arteriolar diameter (pixels) & $18.36(18.14-18.58)$ & $17.90(17.63-18.17)$ & 0.011 \\
\hline Arteriolar LDR & $13.02(12.59-13.47)$ & $13.68(13.12-14.26)$ & 0.073 \\
\hline Bifurcation optimality deviation & $0.044(0.039-0.048)$ & $0.056(0.050-0.063)$ & 0.001 \\
\hline \multicolumn{4}{|c|}{ Retinal variables adjusted for age, sex, ranked $\mathrm{SBP}^{\mathrm{a}}$ and fasting glucose $\mathrm{e}^{\mathrm{b}}$} \\
\hline Arteriolar diameter (pixels) & $18.2(18.0-18.5)$ & $18.1(17.8-18.3)$ & 0.33 \\
\hline Arteriolar LDR & $13.3(12.8-13.7)$ & $13.3(12.8-13.9)$ & 0.93 \\
\hline Bifurcation optimality deviation & $0.044(0.039-0.049)$ & $0.055(0.049-0.062)$ & 0.002 \\
\hline
\end{tabular}

Values are $\%(n)$, means $\pm \mathrm{SD}$ or medians (25th, 75th percentiles), unless otherwise stated

$p$ values were calculated by Student's $t$ test or Mann-Whitney tests for continuous data and by $\chi^{2}$ for categorical data

${ }^{a}$ Treated hypertension allocated to top rank of SBP. SBP and DBP measures are medians (33rd, 67th percentiles)

${ }^{\mathrm{b}}$ Means $(95 \% \mathrm{CI})$

DBP, diastolic BP

There was a significant interaction between BP and diabetes in association with arteriolar diameter in both ethnic groups (age- and sex-adjusted $p$-value for interaction, diabetes $\times \mathrm{SBP}=0.011$ ), such that there was a significant negative and linear gradient in arteriolar diameter across categories of SBP in people without diabetes, but not in those with diabetes (adjusted $\beta$-coefficients for SBP: Europeans: $-0.42, p=0.002$ vs $0.17, p=0.69$, African Caribbeans: $-0.35, p=0.023$ vs $0.01, p=0.96$ (Fig. 1). This interaction was unaltered on adjustment for fasting glucose levels. A similar interaction was observed for arteriolar LDR.

\section{Discussion}

In this population-based study of British people of AfricanCaribbean and European descent, we have demonstrated for the first time that specific quantitative measures of retinal microvascular structure differ by ethnicity and diabetes status.

Compared with Europeans, the relationship between arteriolar diameters at bifurcations was less optimal in African Caribbeans. We interpret the less optimal arteriolar bifurcations observed in African Caribbeans as indicative of impaired microvascular endothelial function in the cerebrovascular microcirculation $[8,9]$, and suggest that this might play a causal role in their elevated risk of stroke. Neither diabetes nor BP explained the ethnic difference in bifurcation optimality.
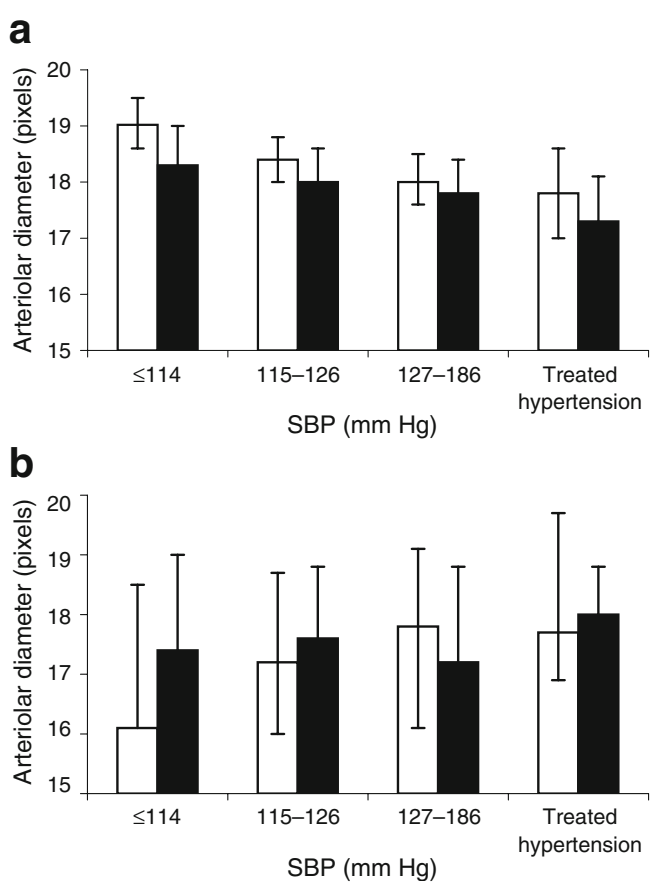

Fig. 1 Arteriolar diameters by ethnicity, tertiles of SBP and diabetes status (age- and sex-adjusted). Means and 95\% CIs. a People without diabetes. White bars, Europeans $(n=292), \beta$-coefficient $-0.42, p=0.002$; black bars, African Caribbeans $(n=161), \beta$-coefficient $-0.35, p=0.023$. b People with diabetes. White bars, Europeans $(n=30), \beta$-coefficient $0.17, p=0.69$; black bars, African Caribbeans $(n=51), \beta$-coefficient $0.01, p=0.96$. $\beta$-Coefficients are for linear trend across SBP categories 
In contrast, arteriolar narrowing in African Caribbeans was explained by higher BPs. In our study, arteriolar narrowing was strongly associated with BP in people without diabetes in both ethnic groups, but this association was lost in people with diabetes. This is similar to the findings of the Cardiovascular Health Study, where relationships between BP and generalised arteriolar narrowing were weaker in diabetic than in non-diabetic individuals [10]. Reasons for this attenuation of the relationship between LDR and BP in diabetes are not known. The reduction of arteriolar diameter increases resistance to blood flow but also serves to protect the capillary bed from the potentially damaging effects of elevated systemic BP. We hypothesise that the increase in arteriolar narrowing in response to elevated BP is impaired in insulin-resistant states, so that no association between LDR and BP is observed. Diabetes is well recognised to result in loss of autoregulation in the retinal circulation, and insulin resistance is also associated with altered cerebrovascular autoregulation. These observations provide a plausible mechanism for the greatly elevated stroke mortality rates in African Caribbeans with diabetes.

Limitations Limitations of the study include $28 \%$ and $16 \%$ of African Caribbeans and Europeans with unanalysable images. It is conceivable that people with more severe retinal disease would be more likely to have unanalysable images and that this might vary by ethnicity. However, there was nothing to suggest that this was the case and the baseline characteristics of the group with analysable images were similar to those of the whole study group; in any case such a scenario would tend to result in underestimation of between group differences.

We adjusted for the effects of antihypertensive medications by ranking people receiving treatment for hypertension as if they had the highest levels of BP; however, similar effects were observed when we adjusted our models for BP without taking medication use into account and when we excluded people receiving antihypertensive medicationsthis suggests that the effects of BP are independent of treatment.

Although we did not detect any significant interactions between ethnicity and other measured risk factors including sex, the study was not sufficiently powered to detect small interactions. However, effects of missed interactions are again likely to lead to underestimation of ethnic group differences.

Further limitations relate to the cross-sectional nature of the study. It is not possible to draw conclusions as to whether $\mathrm{BP}$ and glucose homeostasis are on the causal pathway to the observed retinal vascular findings, or whether suboptimal microvascular structure and function precede impaired glucose homeostasis and hypertension.
By focusing on two a priori primary outcome measures, we attempted to avoid multiple statistical testing in our main analyses, but acknowledge that the strength of associations between LDR, diabetes and ethnicity are relatively weak.

However, these associations offer a plausible explanation for at least part of the ethnic disparity in stroke risk and lead us to hypothesise that cerebral autoregulation and structural remodelling might be adversely affected in the presence of diabetes in both ethnic groups. Microvascular endothelial dysfunction, together with impaired arteriolar structure and function in association with diabetes, presents a possible explanation for the increased risk of stroke experienced by people of African-Caribbean origin. Prospective evidence is required to substantiate these hypotheses.

Acknowledgements The Brent Study was funded by the UK Medical Research Council. These analyses were funded by Diabetes UK. We thank J. Williams and K.-M. Tang for grading the retinal images.

Duality of interest The authors declare that there is no duality of interest associated with this manuscript.

\section{References}

1. Wild SH, Fischbacher C, Brock A, Griffiths C, Bhopal R (2007) Mortality from all causes and circulatory disease by country of birth in England and Wales 2001-2003. J Public Health (Oxf) 29:191-198

2. White H, Boden-Albala B, Wang C et al (2005) Ischemic stroke subtype incidence among whites, blacks, and Hispanics: the Northern Manhattan Study. Circulation 111:1327-1331

3. Wong TY, Klein R, Duncan BB et al (2003) Racial differences in the prevalence of hypertensive retinopathy. Hypertension 41:1086-1091

4. Stanton AV, Mullaney P, Mee F, O’Brien ET, O’Malley K (1995) A method of quantifying retinal microvascular alterations associated with blood pressure and age. J Hypertens 13:41-48

5. Chapman N, Dell'Omo G, Sartini MS et al (2002) Peripheral vascular disease is associated with abnormal arteriolar diameter relationships at bifurcations in the human retina. Clin Sci (Lond) 103:111-116

6. Witt N, Wong TY, Hughes AD et al (2006) Abnormalities of retinal microvascular structure and risk of mortality from ischemic heart disease and stroke. Hypertension 47:975-981

7. Chaturvedi N, McKeigue PM, Marmot MG (1993) Resting and ambulatory blood pressure differences in Afro-Caribbeans and Europeans. Hypertension 22:90-96

8. Murray C (1926) The physiological principle of minimum work applied to the angle of branching of arteries. J Gen Physiol 9:835841

9. Griffith TM, Edwards DH, Davies RLI, Harrison TJ, Evans KT (1987) EDRF coordinates the behaviour of vascular resistance vessels. Nature 329:442-445

10. Wong TY, Hubbard LD, Klein R et al (2002) Retinal microvascular abnormalities and blood pressure in older people: the Cardiovascular Health Study. Br J Ophthalmol 86:10071013 